Article

\title{
Second Order Semilinear Volterra-Type Integro-Differential Equations with Non-Instantaneous Impulses
}

\author{
Mouffak Benchohra ${ }^{1,2}$, Noreddine Rezoug ${ }^{1}$, Bessem Samet ${ }^{2}$ and Yong Zhou ${ }^{3,4, *(\mathbb{D})}$ \\ 1 Laboratory of Mathematics, Djillali Liabes University of Sidi Bel-Abbes, P.O. Box 89, \\ Sidi Bel Abbes 22000, Algeria; benchohra@yahoo.com (M.B.); noreddinerezoug@yahoo.fr (N.R.) \\ 2 Department of Mathematics, College of Science, King Saud University, P.O. Box 2455, \\ Riyadh 11451, Saudi Arabia; bsamet@ksu.edu.sa \\ 3 Faculty of Information Technology, Macau University of Science and Technology, Macau 999078, China \\ 4 Faculty of Mathematics and Computational Science, Xiangtan University, Xiangtan 411105, China \\ * Correspondence: yzhou@xtu.edu.cn
}

Received: 18 August 2019; Accepted: 12 November 2019; Published: 20 November 2019

check for updates

\begin{abstract}
We consider a non-instantaneous system represented by a second order nonlinear differential equation in a Banach space $E$. We use the family of linear bounded operators introduced by Kozak, Darbo fixed point method and Kuratowski measure of noncompactness. A new set of sufficient conditions is formulated which guarantees the existence of the solution of the non-instantaneous system. An example is also discussed to illustrate the efficiency of the obtained results.
\end{abstract}

Keywords: second order differential equations; mild solution; non-instantaneous impulses; Kuratowski measure of noncompactness; Darbo fixed point

\section{Introduction}

The aim of this paper is to establish a result of the existence of mild solution for a class of the non-autonomous second order nonlinear differential equation with non-instantaneous impulses described in the form

$$
\left\{\begin{array}{l}
y^{\prime \prime}(t)=A(t) y(t)+f\left(t, y(t), \int_{0}^{t} g(t, s, y(s)) d s\right), t \in\left(s_{i}, t_{i+1}\right], i=0, \cdots, N, \\
y(t)=\gamma_{i}\left(t, y\left(t_{i}^{-}\right)\right), \quad t \in\left(t_{i}, s_{i}\right], \quad i=1, \cdots, N \\
y^{\prime}(t)=\zeta_{i}\left(t, y\left(t_{i}^{-}\right)\right), \quad t \in\left(t_{i}, s_{i}\right], \quad i=1, \cdots, N \\
y(0)=y_{0}, y^{\prime}(0)=y_{1},
\end{array}\right.
$$

In this text, $E$ is a reflexive Banach space endowed with a norm $|\cdot|, J=[0, a], 0=s_{0}<t_{1}<s_{1}<$ $t_{2}, \cdots, t_{N}<s_{N}<t_{N+1}=a<\infty$. We consider in problem (1) that $y \in C\left(\left(s_{i}, t_{i+1}\right), E\right), i=0,1, \cdots, N$. The functions $\gamma_{i}\left(t, y\left(t_{i}^{-}\right)\right)$and $\zeta_{i}\left(t, y\left(t_{i}^{-}\right)\right)$represent noninstantaneous impulses during the intervals $\left(t_{i}, s_{i}\right], i=1, \cdots, N$, so impulses at $t_{i}^{-}$have some duration, namely on intervals $\left(t_{i}, s_{i}\right]$. Further, $A(t): D(A(t)) \subset E \rightarrow E$ is a closed linear operator which generates a evolution system $\{S(t, s)\}_{(t, s) \in D}$ of linear bounded operators , $f: J \times E \times E \rightarrow E, g \in C(D \times E, E), D=\{(t, s) \in J \times J: s \leq t\}$ and $y_{0}$, $y_{1}$ are given elements of $E$.

The theory and application of integrodifferential equations are important subjects in applied mathematics, see, for example [1-8] and recent development of the topic, see the monographs of [9]. In recent times there have been an increasing interest in studying the abstract autonomous second 
order, see for example [10-14]. Useful for the study of abstract second order equations is the existence of an evolution system $S(t, s)$ for the homogenous equation

$$
y^{\prime \prime}(t)=A(t) y(t), \text { for } t \geq 0 .
$$

For this purpose there are many techniques to show the existence of $S(t, s)$ which has been developed by Kozak [15]. In many problems, such as the transverse motion of an extensible beam, the vibration of hinged bars and many other physical phenomena, we deal with the second-order abstract differential equations in the infinite dimensional spaces. On the other hand, recently there exists an extensive literature for the non-autonomous second order see, for example, [16-22].

The dynamics of many evolving processes are subject to abrupt changes such as shocks, harvesting, and natural disaster. These phenomena involve short term perturbations from continuous and smooth dynamics, whose duration is negligible in comparison with the duration of an entire evolution. Particularly, the theory of instantaneous impulsive equations have wide applications in control, mechanics, electrical engineering, biological and medical fields. Recently, Hernandez et al. [23] use first time not instantaneous impulsive condition for semi-linear abstract differential equation of the form

$$
\left\{\begin{array}{l}
y^{\prime}(t)=A y(t)+f(t, y(t)), t \in\left(s_{i}, t_{i+1}\right], i=0, \cdots, N, \\
y(t)=g_{i}(t, y(t)), \\
y(0)=y_{0},
\end{array} \quad t \in\left(t_{i}, s_{i}\right], i=1, \cdots, N,\right.
$$

and introduced the concepts of mild and classical solution. Wang and Fečkan have changed the conditions $y(t)=g_{i}(t, y(t))$ in (3) as follows

$$
y(t)=g_{i}\left(t, y\left(t_{i}^{+}\right)\right), \quad t \in\left(t_{i}, s_{i}\right], i=1, \cdots, N .
$$

Of course then $y\left(t_{i}^{+}\right)=g_{i}\left(t, y\left(t_{i}^{-}\right)\right)$, where $y\left(t_{i}^{+}\right)$and $y\left(t_{i}^{-}\right)$represent respectively the right and left limits of $y(t)$ at $t=t_{i}$. Motivated by above remark, Wang and Fečkan [24] have shown existence, uniqueness and stability of solutions of such general class of impulsive differential equations. To learn more about this kind of problems, we refer [25-34].

To deal with the above mentioned issues, we investigate necessary and sufficient conditions for the existence of a mild solution of system (1). By virtue of the theory of measure of noncompactness associated with Darbo's and Darbo-Sadovskii's fixed point theorem. This technique was considered by Banas and Goebel [35] and subsequently used in many papers; see, for example, [33,36-39].

A brief outline of this paper is given:. Some preliminaries are presented in Section 2. Section 3, we obtain necessary and sufficient conditions for System (1). An Appropriate example is given to illustrate our results.

\section{Basic Definitions and Preliminaries}

In this section, we review some basic concepts, notations, and properties needed to establish our main results.

Denote by $C(J, E)$ the space of all continuous $E$-valued functions on interval $J$ which is a Banach space with the norm

$$
\|y\|=\sup _{t \in J}|y(t)| .
$$

To treat the impulsive conditions, we define the space of piecewise continuous functions

$$
\begin{aligned}
& P C(J, E)=\left\{y: J \rightarrow E: y \in C\left(\left[0, t_{1}\right] \cup\left(t_{k}, s_{k}\right] \cup\left(s_{k}, t_{k+1}\right], E\right), k=1, \ldots, N\right. \\
& \text { and there exist } y\left(t_{k}^{-}\right), y\left(t_{k}^{+}\right), y\left(s_{k}^{-}\right) \text {and } y\left(s_{k}^{+}\right) k=1, \ldots, N \text { with } y\left(t_{k}^{-}\right)=y\left(t_{k}\right) \\
& \text { and } \left.y\left(s_{k}^{-}\right)=y\left(s_{k}\right)\right\} .
\end{aligned}
$$

It can be easily proved that $P C(J, E)$ is a Banach space endowed with 


$$
\|y\|_{P C}=\sup _{t \in J}|y(t)|
$$

For a positive number $R$, let

$$
B_{R}=\left\{y \in P C(J, E):\|y\|_{P C} \leq R\right\} .
$$

be a bounded set in $P C(J, E)$.

$L^{r}(J, E)$ denotes the space of $E$-valued Bochner functions on $[0, a]$ with the norm

$$
\|y\|_{L^{r}}=\left(\int_{0}^{a}|y(t)|^{r} d t\right)^{\frac{1}{r}}, \quad r \geq 1 .
$$

$B(E)$ the Banach space of bounded linear operators from $E$ into $E$.

First we recall the concept of the evolution operator $S(t, s)$ for problem (2), introduced by Kozak in [15] and recently used by Henríquez, Poblete and Pozo in [20].

Definition 1. Let $S: D \rightarrow B(E)$. The family is said to be an evolution operator generated by the family $\{A(t): t \in J\}$ if the following conditions are satisfied [15]:

$\left(e_{1}\right)$ For each $y \in E$ the function $S(\cdot, \cdot) y: J \times J \rightarrow E$ is of class $C^{1}$ and

(i) for each $t \in J, S(t, t)=0$,

(ii) for all $(t, s) \in D$ and for each $y \in E$,

$$
\left.\frac{\partial}{\partial t} S(t, s) y\right|_{t=s}=y,\left.\frac{\partial}{\partial s} S(t, s) y\right|_{t=s}=-y .
$$

$\left(e_{2}\right)$ For each $(t, s) \in D$, if $y \in D(A(t))$, then $\frac{\partial}{\partial s} S(t, s) y \in D(A(t))$, the map $(t, s) \longmapsto S(t, s) y$ is of class $C^{2}$ and

(i) $\frac{\partial^{2}}{\partial t^{2}} S(t, s) y=A(t) S(t, s) y$,

(ii) $\frac{\partial^{2}}{\partial s_{\partial^{2}}^{2}} S(t, s) y=S(t, s) A(s) y$,

(iii) $\left.\frac{\partial^{2}}{\partial s \partial t} S(t, s) y\right|_{t=s}=0$.

$\left(e_{3}\right)$ For all $(t, s) \in D$, if $y \in D(A(t))$, then $\frac{\partial}{\partial s} S(t, s) y \in D(A(t))$. Moreover, there exist $\frac{\partial^{3}}{\partial t^{2} \partial s} S(t, s) y$, $\frac{\partial^{3}}{\partial s^{2} \partial t} S(t, s) y$ and

(i) $\frac{\partial^{3}}{\partial t^{2} \partial s} S(t, s) y=A(t) \frac{\partial}{\partial s} S(t, s) y$,

(ii) $\frac{\partial^{3}}{\partial s^{2} \partial t} S(t, s) y=\frac{\partial}{\partial t} S(t, s) A(s) y$,

and for all $y \in D(A)$ the function $(t, s) \longmapsto A(t) \frac{\partial}{\partial s} S(t, s) y$ is continuous in $D$.

Definition 2. A function $f: J \times E \times E \rightarrow E$ is said to be a Carathéodory function if it satisfies:

(i) $t \rightarrow f(t, u, v)$ is measurable for each $u, v \in E \times E$,

(ii) $(u, v) \rightarrow f(t, u, v)$ is continuous for almost each $t \in J$.

For $W$, a nonempty subset of $E$, we denote by $\bar{W}$ and ConvW the closure and the closed convex hull of $W$, respectively. Finally, the standard algebraic operations on sets are denoted by $a W$ and $Y+W$, respectively. Now, we recall some basic definitions and properties about Kuratowski measure of noncompactness that will be used in the proof of our main results. 
Definition 3. [35] The Kuratowski measure of noncompactness $\alpha_{E}(\cdot)$ defined on bounded set $W$ of Banach space $E$ is

$$
\alpha_{E}(W)=\inf \left\{\varepsilon>0: W=\cup_{i=1}^{n} W_{i} \text { and } \operatorname{diam}\left(W_{i}\right) \leq \varepsilon \text { for } i=1,2, \cdots n\right\}
$$

Some basic properties of $\alpha_{E}(\cdot)$ are given in the following lemma.

Lemma 1. Let $Y$ and $W$ be bounded sets of $E$ and a be a real number [35]. The Kuratowski measure of noncompactness satisfies some properties:

$\left(p_{1}\right) W$ is pre-compact if and only if $\alpha_{E}(W)=0$,

$\left(p_{2}\right) \alpha_{E}(\bar{W})=\alpha_{E}(W)$,

$\left(p_{3}\right) \alpha_{E}(Y) \leq \alpha_{E}(W)$ when $Y \subset W$,

$\left(p_{4}\right) \alpha_{E}(Y+W) \leq \alpha_{E}(Y)+\alpha_{E}(W)$

$\left(p_{5}\right) \alpha_{E}(a W)=|a| \alpha_{E}(W)$ for any $a \in \mathbf{R}$,

$\left(p_{6}\right) \alpha_{E}(\operatorname{Conv} W)=\alpha_{E}(W)$.

The map $Q: X \subset E \rightarrow E$ is said to be a $\alpha$-contraction if there exists a positive constant $\lambda<1$ such that $\alpha_{E}(Q(W)) \leq \lambda \alpha_{E}(W)$ for any bounded closed subset $W \subset E$.

Lemma 2. [40] Let $E$ be a Banach space, $W \subset E$ be bounded. Then there exists a countable set $W_{0} \subset W$, such that

$$
\alpha_{E}(W) \leq 2 \alpha_{E}\left(W_{0}\right)
$$

Lemma 3. [41] Let $E$ be a Banach space, $-\infty<a_{1}<a_{2}<+\infty$ for constants, and let $W=\left\{y_{n}\right\} \subset$ $P C\left(\left[a_{1}, a_{2}\right], E\right)$, be a bounded and countable set. Then $\alpha_{E}(W(t))$ is Lebesgue integral on $\left[a_{1}, a_{2}\right]$, and

$$
\alpha_{E}\left(\left\{\int_{a_{1}}^{a_{2}} y_{n}(t) d t: n \in \mathbb{N}\right\}\right) \leq 2 \int_{a_{1}}^{a_{2}} \alpha_{E}(W(t)) d t
$$

Denote by $\alpha_{P C}$ the Kuratowski measure of noncompactness of $P C(J, E)$. Before proving the existence results, we need the following Lemmas.

Lemma 4. [35] If $W \subset P C(J ; E)$ is bounded, then $\alpha_{E}(W(t)) \leq \alpha_{P C}(W)$, for all $t \in J$; here $W(t)=$ $\{y(t) ; y \in W \subset E\}$. Furthermore if $W$ is equicontinuous on $J$, then $\alpha_{E}(W(t))$ is continuous on $J$ and

$$
\alpha_{P C}(W)=\sup _{t \in J} \alpha_{E}(W(t))
$$

Lemma 5. [42] Let $E, F$ be Banach spaces. If the map $\Psi: \mathcal{D}(\Psi) \subset E \rightarrow F$ is Lipschitz continuous with constant $k$, then $\alpha_{E}(\Psi(W)) \leq k \alpha_{E}(W)$ for any bounded subset $W \subset \mathcal{D}(\Psi)$.

Theorem 1. (Darbo) [43] Assume that $W$ is a non-empty, closed and convex subset of a Banach space $E$ and $0 \in W$. Let $Q: W \rightarrow W$ be a continuous mapping and $\alpha_{E}$-contraction. If the set $\{y \in W: y=\lambda Q y\}$ is bounded for $0<\lambda<1$, then the map $Q$ has at least one fixed point in $W$.

Theorem 2. (Darbo-Sadovskii) [35] Assume that W is a non-empty, closed, bounded, and convex subset of a Banach space $E$. Let $Q: W \rightarrow W$ be a continuous mapping and $\alpha_{E}$-contraction. Then the map $Q$ has at least one fixed point in $W$.

\section{Existence Results}

In this section, we discuss the existence of mild solutions for system (1). Firstly, let us propose the definition of the mild solution of system (1). 
Definition 4. A function $y \in P C(J, E)$ is said to be a mild solution to the system (1), if it satisfies the following relations:

$$
y(0)=y_{0}, y^{\prime}(0)=y_{1}
$$

the non-instantaneous conditions

$$
y(t)=\gamma_{i}\left(t, y\left(t_{i}^{-}\right)\right), \quad y^{\prime}(t)=\zeta_{i}\left(t, y\left(t_{i}^{-}\right)\right), \quad t \in\left(t_{i}, s_{i}\right],
$$

and $y$ is the solution of the following integral equations

$$
y(t)=\left\{\begin{aligned}
-\frac{\partial}{\partial s} S(t, 0) y_{0}+S(t, 0) y_{1} & \\
& +\int_{0}^{t} S(t, s) f\left(s, y(s), \int_{0}^{s} g(s, \tau, y(\tau)) d \tau\right) d s, \quad t \in\left[0, t_{1}\right] \\
-\frac{\partial}{\partial s} S\left(t, s_{i}\right) \gamma_{i}\left(s_{i}, y\left(t_{i}^{-}\right)\right)+S\left(t, s_{i}\right) \zeta_{i}\left(s_{i}, y\left(t_{i}^{-}\right)\right) & \\
& +\int_{s_{i}}^{t} S(t, s) f\left(s, y(s), \int_{0}^{s} g(s, \tau, y(\tau)) d \tau\right) d s, \quad t \in\left(s_{i}, t_{i+1}\right] .
\end{aligned}\right.
$$

In this manuscript, we list the following hypotheses:

$\left(H_{1}\right)$ There exist a pair of constants $M \geq 1$ and $\delta>0$, such that

$$
\|S(t, s)\|_{B(E)} \leq M e^{-\delta(t-s)} \text { for any }(t, s) \in D .
$$

$\left(H_{2}\right)$ There exists a constant $\tilde{M}>0$ such that:

$$
\left\|\frac{\partial}{\partial s} S(t, s)\right\|_{B(E)} \leq \tilde{M} e^{-\delta(t-s)},(t, s) \in D .
$$

$\left(H_{3}\right) f: J \times E \times E \rightarrow E$ is of Carathéodory type and satisfies:

(a) There exist $\Theta_{f} \in L^{r}\left(J, \mathbb{R}^{+}\right), r \in[1, \infty)$ and a continuous nondecreasing function $\psi:[0, \infty) \rightarrow$ $(0, \infty)$ such that:

$$
|f(t, y, z)| \leq \Theta_{f}(t) \psi(|y|+|z|) \text { for a.a } t \in J \text { and each } y, z \in E .
$$

(b) There exist integrable functions $\sigma, \varrho: J \rightarrow \mathbb{R}^{+}$, such that:

$$
\begin{gathered}
\alpha_{E}\left(f\left(t, W_{1}, W_{2}\right)\right) \leq \sigma(t) \alpha_{E}\left(W_{1}\right)+\varrho(t) \alpha_{E}\left(W_{2}\right) \\
\text { for a.a } t \in J \text { and } W_{1}, W_{2} \subset E .
\end{gathered}
$$

$\left(H_{4}\right) g: D \times E \rightarrow E$ is a continuous function that satisfies:

(a) There exist $\Theta_{g} \in L^{1}\left(J, \mathbb{R}^{+}\right)$, and a continuous nondecreasing function $\varphi:[0, \infty) \rightarrow(0, \infty)$ such that:

$$
|g(t, s, y)| \leq \Theta_{g}(t) \varphi(|y|) \text { for a.a }(t, s) \in D \text { and each } y \in E .
$$

(b) There exists constant $K^{*}>0$, such that

$$
\alpha_{E}(g(t, s, W)) \leq K^{*} \alpha_{E}(W) \text { for a.a }(t, s) \in D \text { and } W \subset E .
$$

$\left(H_{5}\right)$ The functions $\gamma_{i}:\left(t_{i}, s_{i}\right] \times E \rightarrow E, i=1, \cdots, N$, are continuous, and they satisfy the following conditions:

(a) there exist positive constants $c_{i}, i=1, \cdots, N$ such that

$$
\left|\gamma_{i}\left(t, y_{2}\right)-\gamma_{i}\left(t, y_{1}\right)\right| \leq c_{i}\left|y_{2}-y_{1}\right| \text { for a.a } t \in\left(t_{i}, s_{i}\right] \text { and each } y_{1}, y_{2} \in E \text {. }
$$


(b) there exist positive constants $d_{i}$, such that

$$
d_{i}=\sup _{t \in\left[t_{i}, s_{i}\right]} \gamma_{i}(t, 0)
$$

$\left(H_{6}\right)$ The functions $\zeta_{i}:\left(t_{i}, s_{i}\right] \times E \rightarrow E, i=1, \cdots, N$, are continuous, and satisfy the following conditions:

(a) There exist constants $e_{i}, l_{i}>0, i=1, \cdots, N$ such that

$$
\left|\zeta_{i}(t, y)\right| \leq e_{i}|y|+l_{i} \text { for a.a } t \in\left(t_{i} ; s_{i}\right] \text { and each } y \in E .
$$

(b) There exists constants $\bar{k}_{i}>0, i=1, \cdots, N$ such that

$$
\alpha_{E}\left(\zeta_{i}(t, W)\right) \leq \bar{k}_{i} \alpha_{E}(W) \text { for a.a } t \in\left(t_{i}, s_{i}\right] \text { and anyW } \subset E .
$$

$\left(H_{7}\right)$

$$
\max _{1 \leq i \leq N}\left(k_{i}, 1\right)\left(\max _{1 \leq i \leq N}\left(\tilde{M} k_{i}+M \bar{k}_{i}\right)+2 M\left(\|\sigma\|_{L^{1}}+2 K^{*} a\|\varrho\|_{L^{1}}\right)\right)<1 .
$$

Remark 1. From Lemma 5 and $\left(H_{5}\right)$, there exist constants $k_{i}>0$, such that

$$
\alpha_{E}\left(\gamma_{i}(t, W)\right) \leq k_{i} \alpha_{E}(W) \text { for a.a } t \in\left(t_{i}, s_{i}\right] \text { and each } y \in E
$$

Theorem 3. Under the assumptions $\left(H_{1}\right)-\left(H_{7}\right)$, the system (1) has at least one mild solution on J, provided that

$$
\int_{0}^{a} \max \left(\bar{M} \Theta_{f}(s), \Theta_{g}(s)\right) d s \leq \int_{m_{i}}^{\infty} \frac{d s}{\psi(s)+\varphi(s)}, i=2,3 \cdots N
$$

with

$$
\bar{M}=\max _{2 \leq i \leq N}\left\{\frac{M}{1-L_{1}}, \frac{M}{1-L_{i}}, \frac{M c_{i}}{1-L_{i-1}}\right\}
$$

and

$$
m_{i}=\frac{d_{i}}{1-L_{i-1}}+\max _{2 \leq i \leq N}\left\{\tilde{M}\left|y_{0}\right|+M\left|y_{1}\right|, \frac{\tilde{M} d_{1}}{1-L_{1}}+\frac{M l_{1}}{1-L_{1}}, \frac{\tilde{M} d_{i}}{1-L_{i}}+\frac{M l_{i}}{1-L_{i}}, \frac{\tilde{M} c_{i} d_{i-1}}{1-L_{i-1}}+\frac{M c_{i} l_{i-1}}{1-L_{i-1}}\right\},
$$

where

$$
L_{i}=\tilde{M} c_{i}+M e_{i}<1
$$

Proof. Define the mapping $\Lambda: P C(J, E) \rightarrow P C(J, E)$ by

$$
(\Lambda y)(t)=\left\{\begin{array}{c}
\gamma_{i}\left(t,-\frac{\partial}{\partial s} S\left(t, s_{i}\right) \gamma_{i-1}\left(s_{i-1}, y\left(t_{i-1}^{-}\right)\right)+S\left(t, s_{i-1}\right) \zeta_{i-1}\left(s_{i-1}, y\left(t_{i-1}^{-}\right)\right)\right. \\
\left.\quad+\int_{s_{i-1}}^{t_{i}} S(t, s) f\left(s, y(s), \int_{0}^{s} g(s, \tau, y(\tau)) d \tau\right) d s\right), \quad t \in\left(t_{i}, s_{i}\right] \\
-\frac{\partial}{\partial s} S(t, 0) y_{0}+S(t, 0) y_{1} \\
\quad+\int_{0}^{t} S(t, s) f\left(s, y(s), \int_{0}^{s} g(s, \tau, y(\tau)) d \tau\right) d s, \quad t \in\left[0, t_{1}\right] \\
-\frac{\partial}{\partial s} S\left(t, s_{i}\right) \gamma_{i}\left(s_{i}, y\left(t_{i}^{-}\right)\right)+S\left(t, s_{i}\right) \zeta_{i}\left(s_{i}, y\left(t_{i}^{-}\right)\right) \\
\quad+\int_{s_{i}}^{t} S(t, s) f\left(s, y(s), \int_{0}^{s} g(s, \tau, y(\tau)) d \tau\right) d s, \quad t \in\left(s_{i}, t_{i+1}\right] .
\end{array}\right.
$$

It is obvious that the fixed point of $\Lambda$ is the mild solution of (1). We shall show that $\Lambda$ satisfies the assumptions of Theorem 1 . The proof will be given in four steps. 
Step 1. A priori bounds.

Let $\lambda \in(0,1)$ and let $y \in Y$ be a possible solution of $y=\lambda \Lambda(y)$ for some $0<\lambda<1$. Thus,

Case 1. For each $t \in\left[0, t_{1}\right]$, we get

$$
y(t)=-\lambda \frac{\partial}{\partial s} S(t, 0) y_{0}+\lambda S(t, 0) y_{1}+\lambda \int_{0}^{t} S(t, s) f\left(s, y(s), \int_{0}^{s} g(s, \tau, y(\tau)) d \tau\right) d s .
$$

Then

$$
\begin{aligned}
|y(t)| & \leq\left\|\frac{\partial}{\partial s} S(t, 0)\right\|_{B(E)}\left|y_{0}\right|+\|S(t, 0)\|_{B(E)}\left|y_{1}\right| \\
& \left.+\int_{0}^{t}\|S(t, s)\|_{B(E)} \Theta_{f}(s) \psi\left(|y(s)|+\int_{0}^{s} \Theta_{g}(\tau) \varphi(|y(\tau)|)\right) d \tau\right) d s \\
& \leq \tilde{M}\left|y_{0}\right| e^{-\delta t}+M\left|y_{1}\right| e^{-\delta t} \\
& +\int_{0}^{t} M e^{-\delta(t-s)} \Theta_{f}(s) \psi\left(|y(s)|+\int_{0}^{s} \Theta_{g}(\tau) \varphi(|y(\tau)|) d \tau\right) d s \\
& \leq\left(\tilde{M}\left|y_{0}\right|+M\left|y_{1}\right|\right) e^{-\delta t} \\
& +\int_{0}^{t} M e^{-\delta(t-s)} \Theta_{f}(s) \psi\left(|y(s)|+\int_{0}^{s} \Theta_{g}(\tau) \varphi(|y(\tau)|) d \tau\right) d s
\end{aligned}
$$

Case 2. For each $t \in\left(s_{i}, t_{i+1}\right]$, we have

$$
\begin{aligned}
y(t) & =-\lambda \frac{\partial}{\partial s} S\left(t, s_{i}\right) \gamma_{i}\left(s_{i}, y\left(s_{i}\right)\right)+\lambda S\left(t, s_{i}\right) \zeta_{i}\left(s_{i}, y\left(s_{i}\right)\right) \\
& +\lambda \int_{s_{i}}^{t} S(t, s) f\left(s, y(s), \int_{0}^{s} g(s, \tau, y(\tau)) d \tau\right) d s
\end{aligned}
$$

then

$$
\begin{aligned}
|y(t)| & \leq\left\|\frac{\partial}{\partial s} S\left(t, s_{i}\right)\right\|_{B(E)}\left|\gamma_{i}\left(s_{i}, y\left(s_{i}\right)\right)\right|+\left\|S\left(t, s_{i}\right)\right\|_{B(E)}\left|\zeta_{i}\left(s_{i}, y\left(s_{i}\right)\right)\right| \\
& \left.+\int_{s_{i}}^{t}\|S(t, s)\|_{B(E)} \Theta_{f} \psi\left(|y(s)|+\int_{0}^{s} \Theta_{g}(\tau) \varphi(|y(\tau)|)\right) d \tau\right) d s \\
& \leq \tilde{M} c_{i}\left|y\left(s_{i}\right)\right| e^{-\delta\left(t-s_{i}\right)}+\tilde{M} d_{i} e^{-\delta\left(t-s_{i}\right)} \\
& +M e_{i}\left|y\left(s_{i}\right)\right| e^{-\delta\left(t-s_{i}\right)}+M l_{i} e^{-\delta\left(t-s_{i}\right)} \\
& +\int_{s_{i}}^{t} M e^{-\delta(t-s)} \Theta_{f}(s) \psi\left(|y(s)|+\int_{0}^{s} \Theta_{g}(\tau) \varphi(|y(\tau)|) d \tau\right) d s . \\
& \leq \tilde{M} c_{i}\left|y\left(s_{i}\right)\right|+\tilde{M} d_{i} e^{-\delta\left(t-s_{i}\right)} \\
& +M e_{i}\left|y\left(s_{i}\right)\right|+M l_{i} e^{-\delta\left(t-s_{i}\right)} \\
& +\int_{s_{i}}^{t} M e^{-\delta(t-s)} \Theta_{f}(s) \psi\left(|y(s)|+\int_{0}^{s} \Theta_{g}(\tau) \varphi(|y(\tau)|) d \tau\right) d s .
\end{aligned}
$$

It is easy to see that

$$
\begin{aligned}
\sup _{s \in[0, t]}|y(s)| & \leq\left(\frac{\tilde{M} d_{i} e^{s_{i}}}{1-L_{i}}+\frac{M l_{i} e^{s_{i}}}{1-L_{i}}\right) e^{-\delta t} \\
& +\int_{s_{i}}^{t} \frac{M}{1-L_{i}} e^{-\delta(t-s)} \Theta_{f}(s) \psi\left(\sup _{s \in[0, t]}|y(s)|+\int_{0}^{s} \Theta_{g}(\tau) \varphi\left(\sup _{s \in[0, t]} \mid y(s)\right) \mid d \tau\right) d s .
\end{aligned}
$$

Case 3. For each $t \in\left(s_{i}, t_{i}\right]$, we have, 


$$
\begin{aligned}
|y(t)| & =\lambda \mid \gamma_{i}\left(t,-\frac{\partial}{\partial s} S\left(t, s_{i-1}\right) \gamma_{i-1}\left(s_{i-1}, y\left(t_{i-1}^{-}\right)\right)+S\left(t, s_{i-1}\right) \zeta_{i-1}\left(s_{i-1}, y\left(t_{i-1}^{-}\right)\right)\right. \\
& \left.+\int_{s_{i-1}}^{t_{i}} S(t, s) f\left(s, y(s), \int_{0}^{s} g(s, \tau, y(\tau)) d \tau\right) d s\right) \mid \\
& \leq \lambda \mid \gamma_{i}\left(t,-\frac{\partial}{\partial s} S\left(t, s_{i}\right) \gamma_{i-1}\left(s_{i-1}, y\left(t_{i-1}^{-}\right)\right)+S\left(t, s_{i-1}\right) \zeta_{i-1}\left(s_{i-1}, y\left(t_{i-1}^{-}\right)\right)\right. \\
& \left.+\int_{s_{i-1}}^{t_{i}} S(t, s) f\left(s, y(s), \int_{0}^{s} g(s, \tau, y(\tau)) d \tau\right) d s-\gamma_{i}(t, 0)\right) \mid \\
& +\lambda\left|\gamma_{i}(t, 0)\right| \\
& \leq \lambda c_{i} \mid-\frac{\partial}{\partial s} S\left(t, s_{i}\right) \gamma_{i-1}\left(s_{i-1}, y\left(t_{i-1}^{-}\right)\right)+S\left(t, s_{i-1}\right) \zeta_{i-1}\left(s_{i-1}, y\left(t_{i-1}^{-}\right)\right) \\
& +\int_{s_{i-1}}^{t_{i}} S(t, s) f\left(s, y(s), \int_{0}^{s} g(s, \tau, y(\tau)) d \tau\right) d s \mid \\
& +\lambda d_{i} .
\end{aligned}
$$

This implies

$$
\begin{aligned}
\sup _{s \in[0, t]}|y(s)| & \leq\left(\frac{\tilde{M} c_{i} d_{i-1} e^{s_{i-1}}}{1-L_{i-1}}+\frac{M c_{i} l_{i-1} e^{s_{i-1}}}{1-L_{i-1}}\right) e^{-\delta t}+\frac{d_{i}}{1-L_{i-1}} \\
& +\int_{s_{i-1}}^{t_{i}} \frac{M c_{i}}{1-L_{i-1}} e^{-\delta(t-s)} \Theta_{f}(s) \psi\left(\sup _{\tau \in[0, s]}|y(\tau)|+\int_{0}^{s} \Theta_{g}(\tau) \varphi\left(\sup _{z \in[0, \tau]}|y(z)|\right) d \tau\right) d s .
\end{aligned}
$$

Then, for all $t \in J$, we have

$$
\begin{aligned}
|y(t)| & \leq M_{i}^{*} e^{-\delta t}+\frac{d_{i}}{1-L_{i-1}} \\
& +e^{-\delta t} \int_{0}^{t} \bar{M} e^{\delta s} \Theta_{f}(s) \psi\left(\sup _{\tau \in[0, s]}|y(\tau)|+\int_{0}^{s} \Theta_{g}(\tau) \varphi\left(\sup _{z \in[0, \tau]}|y(z)|\right) d \tau\right) d s .
\end{aligned}
$$

where

$$
M^{*}=\max _{2 \leq i \leq N}\left\{\tilde{M}\left|y_{0}\right|+M\left|y_{1}\right|, \frac{\tilde{M} d_{1} e^{s_{1}}}{1-L_{1}}+\frac{M l_{1} e^{s_{1}}}{1-L_{1}}, \frac{\tilde{M} d_{i} e^{s_{i}}}{1-L_{i}}+\frac{M l_{i} e^{s_{i}}}{1-L_{i}}, \frac{\tilde{M} c_{i} d_{i-1} e^{s_{i-1}}}{1-L_{i-1}}+\frac{M c_{i} l_{i-1} e^{s_{i-1}}}{1-L_{i-1}}\right\} .
$$

Let us take the right-hand side of the above inequality as $\mu(t)$. Then

$$
\begin{gathered}
\mu(0)=M^{*}+\frac{d_{i}}{1-L_{i-1}}, \\
\sup _{s \in[0, t]}|y(s)| \leq \mu(t),
\end{gathered}
$$

and

$$
\begin{aligned}
\mu^{\prime}(t) & \leq-\delta \mu(t)+\bar{M} \Theta_{f}(t) \psi\left(\mu(t)+\int_{0}^{t} \Theta_{g}(s) \varphi(\mu(s)) d s\right) \\
& \leq \bar{M} \Theta_{f}(t) \psi\left(\mu(t)+\int_{0}^{t} \Theta_{g}(s) \varphi(\mu(s)) d s\right) .
\end{aligned}
$$

Let

$$
\beta(t)=\mu(t)+\int_{0}^{t} \Theta_{g}(s) \varphi(\mu(s)) d s
$$

Then

$$
\begin{aligned}
\beta^{\prime}(t) & =\mu^{\prime}(t)+\Theta_{g}(t) \varphi(\mu(t)) \\
& \leq \bar{M} \Theta_{f}(t) \psi(\beta(t))+\Theta_{g}(t) \varphi(\beta(t)) .
\end{aligned}
$$


This implies that

$$
\int_{\beta(0)}^{\beta(t)} \frac{d s}{\psi(s)+\varphi(s)} \leq \int_{m_{i}}^{a} \max \left(\bar{M} \Theta_{f}(s), \Theta_{g}(s)\right) d s<\int_{m_{i}}^{+\infty} \frac{d s}{\psi(s)+\varphi(s)} .
$$

This above inequality implies that there exists a constant $L$ such that $\beta(t) \leq L, t \in J$, and hence $\mu(t) \leq L, t \in J$. Since for every $t \in J,|y(t)| \leq \mu(t)$, we have $\|y\|_{P C} \leq L$.

Step 2. $\Lambda$ is continuous.

Suppose that $\left(y_{n}\right)_{n \in \mathbb{N}}$ is a sequence in $B_{R}$ which converges to $y$ in $B_{R}$ as $n \rightarrow \infty$. By the continuity of nonlinear term $\gamma$ and $\zeta$ with respect to the second argument, for each $s \in J$, we have

$$
\begin{aligned}
& \sup _{s \in J}\left|\gamma_{i}\left(s, y_{n}(s)\right)-\gamma_{i}(s, y(s))\right| \rightarrow 0 \quad \text { as } \quad n \rightarrow \infty, \\
& \sup _{s \in J}\left|\zeta\left(s, y_{n}(s)\right)-\zeta(s, y(s))\right| \rightarrow 0 \quad \text { as } \quad n \rightarrow \infty .
\end{aligned}
$$

By the Carathéodory character of nonlinear term $f$, for each $s \in J$, we have

$$
\left|f\left(s, y_{n}(s), \int_{0}^{s} g\left(s, \tau, y_{n}(\tau)\right) d \tau\right)-f\left(s, y(s), \int_{0}^{s} g(s, \tau, y(\tau)) d \tau\right)\right| \rightarrow 0 \text { as } n \rightarrow \infty .
$$

Case 1. For the interval $\left(s_{i}, t_{i}\right]$, we obtain

$$
\begin{aligned}
& \left|\left(\Lambda y_{n}\right)(t)-(\Lambda y)(t)\right| \\
& \leq \gamma_{i}\left(t,-\frac{\partial}{\partial s} S\left(t, s_{i}\right) \gamma_{i-1}\left(s_{i-1}, y_{n}\left(t_{i-1}^{-}\right)\right)+S\left(t, s_{i-1}\right) \zeta_{i-1}\left(s_{i-1}, y_{n}\left(t_{i-1}^{-}\right)\right)\right. \\
& \left.\quad+\int_{s_{i-1}}^{t_{i}} S(t, s) f\left(s, y_{n}(s), \int_{0}^{s} g\left(s, \tau, y_{n}(\tau)\right) d \tau\right) d s\right) \\
& -\gamma_{i}\left(t,-\frac{\partial}{\partial s} S\left(t, s_{i}\right) \gamma_{i-1}\left(s_{i-1}, y\left(t_{i-1}^{-}\right)\right)+S\left(t, s_{i-1}\right) \zeta_{i-1}\left(s_{i-1}, y\left(t_{i-1}^{-}\right)\right)\right. \\
& \left.\quad+\int_{s_{i-1}}^{t_{i}} S(t, s) f\left(s, y(s), \int_{0}^{s} g(s, \tau, y(\tau)) d \tau\right) d s\right)
\end{aligned}
$$

Since the function $\gamma_{i}$ is continuous and

$$
\begin{aligned}
& \mid-\frac{\partial}{\partial s} S\left(t, s_{i}\right) \gamma_{i-1}\left(s_{i-1}, y_{n}\left(t_{i-1}^{-}\right)\right)+S\left(t, s_{i-1}\right) \zeta_{i-1}\left(s_{i-1}, y_{n}\left(t_{i-1}^{-}\right)\right) \\
& +\int_{s_{i-1}}^{t_{i}} S(t, s) f\left(s, y_{n}(s), \int_{0}^{s} g\left(s, \tau, y_{n}(\tau)\right) d \tau\right) d s+\frac{\partial}{\partial s} s\left(t, s_{i}\right) \gamma_{i-1}\left(s_{i-1}, y\left(t_{i-1}^{-}\right)\right) \\
& +S\left(t, s_{i-1}\right) \zeta_{i-1}\left(s_{i-1}, y\left(t_{i-1}^{-}\right)\right)+\int_{s_{i-1}}^{t_{i}} S(t, s) f\left(s, y(s), \int_{0}^{s} g(s, \tau, y(\tau)) d \tau\right) d s \mid \\
& \leq \tilde{M}\left|\gamma_{i-1}\left(s_{i-1}, y_{n}\left(s_{i-1}\right)\right)-\gamma_{i-1}\left(s_{i}, y\left(s_{i}\right)\right)\right|+M\left|\zeta_{i-1}\left(s_{i-1}, y_{n}\left(s_{i-1}\right)\right)-\zeta_{i-1}\left(s_{i-1}, y\left(s_{i-1}\right)\right)\right| \\
& +M \int_{s_{i-1}}^{t}\left|f\left(s, y_{n}(s), \int_{0}^{s} g\left(s, \tau, y_{n}(\tau)\right) d \tau\right)-f\left(s, y(s), \int_{0}^{s} g(s, \tau, y(\tau)) d \tau\right)\right| d s . \\
& \rightarrow 0, \quad \text { as } n \rightarrow \infty .
\end{aligned}
$$

We can conclude that $\Lambda y_{n} \rightarrow \Lambda y$, as $n \rightarrow+\infty$.

Case 2. For the interval $\left[0, t_{1}\right]$, we obtain

$$
\begin{aligned}
& \left|\left(\Lambda y_{n}\right)(t)-(\Lambda y)(t)\right| \\
& \leq M \int_{0}^{t}\left|f\left(s, y_{n}(s), \int_{0}^{s} g\left(s, \tau, y_{n}(\tau)\right) d \tau\right)-f\left(s, y(s), \int_{0}^{s} g(s, \tau, y(\tau)) d \tau\right)\right| d s \\
& \rightarrow 0, \quad \text { as } n \rightarrow \infty .
\end{aligned}
$$


Case 3. For the interval $\left(s_{i}, t_{i+1}\right]$, we have

$$
\begin{aligned}
& \left|\left(\Lambda y_{n}\right)(t)-(\Lambda y)(t)\right| \\
& \leq \tilde{M}\left|\gamma_{i}\left(s_{i}, y_{n}\left(s_{i}\right)\right)-\gamma_{i}\left(s_{i}, y\left(s_{i}\right)\right)\right|+M\left|\zeta_{i}\left(s_{i}, y_{n}\left(s_{i}\right)\right)-\zeta_{i}\left(s_{i}, y\left(s_{i}\right)\right)\right| \\
& +M \int_{s_{i}}^{t}\left|f\left(s, y_{n}(s), \int_{0}^{s} g\left(s, \tau, y_{n}(\tau)\right) d \tau\right)-f\left(s, y(s), \int_{0}^{s} g(s, \tau, y(\tau)) d \tau\right)\right| d s \\
& \rightarrow 0 \quad \text { as } n \rightarrow \infty .
\end{aligned}
$$

As a consequence of Case $1-3, \Lambda y_{n} \rightarrow \Lambda y$, as $n \rightarrow+\infty$. Hence the $\Lambda$ is continuous.

Step 3. $\Lambda$ is equicontinuous.

Case 1 . For the interval $\left[0, t_{1}\right], 0 \leq \tilde{t}_{1} \leq \tilde{t}_{2} \leq t_{1}$, any $y \in B_{R}$, we have

$$
\begin{aligned}
& \left|(\Lambda y)\left(\tilde{t}_{2}\right)-(\Lambda y)\left(\tilde{t}_{1}\right)\right| \\
& \leq\left\|\frac{\partial}{\partial s} S\left(\tilde{t}_{2}, 0\right)-\frac{\partial}{\partial s} S\left(\tilde{t}_{1}, 0\right)\right\|_{B(E)}\left|y_{0}\right| \\
& +\left\|S\left(\tilde{t}_{2}, 0\right)-S\left(\tilde{t}_{1}, 0\right)\right\|_{B(E)}\left|y_{1}\right| \\
& +\mid \int_{0}^{\tilde{t}_{1}}\left(S\left(\tilde{t}_{2}, s\right)-S\left(\tilde{t}_{1}, s\right)\right) f\left(s, y(s), \int_{0}^{s} g(s, \tau, y(\tau)) d \tau\right) d s \\
& +\int_{\tilde{t}_{1}}^{\tilde{t}_{2}} S\left(\tilde{t}_{2}, \tau\right) f\left(s, y(s), \int_{0}^{s} g(s, \tau, y(\tau)) d \tau\right) d s \mid \\
& \leq \int_{0}^{\tilde{t}_{1}}\left\|S\left(\tilde{t}_{2}, \tau\right)-S\left(\tilde{t}_{1}, \tau\right)\right\|_{B(E)} \Theta_{f}(\tau) \psi\left(|y(s)|+\int_{0}^{s} \Theta_{g}(\tau) \varphi(|y(\tau)|) d \tau\right) d s \\
& +M \int_{\tilde{t}_{1}}^{\tilde{t}_{2}} \Theta_{f}(s) \psi\left(|y(s)|+\int_{0}^{s} \Theta_{g}(\tau) \varphi(|y(\tau)|) d \tau\right) d s .
\end{aligned}
$$

It follows from the Hölder's inequality that

$$
\begin{aligned}
& \left|(\Lambda y)\left(\tilde{t}_{2}\right)-(\Lambda y)\left(\tilde{t}_{1}\right)\right| \\
& \leq\left\|\frac{\partial}{\partial s} S\left(\tilde{t}_{2}, 0\right)-\frac{\partial}{\partial s} S\left(\tilde{t}_{1}, 0\right)\right\|_{B(E)}\left|y_{0}\right| \\
& +\left\|S\left(\tilde{t}_{2}, 0\right)-S\left(\tilde{t}_{1}, 0\right)\right\|_{B(E) \mid}\left|y_{1}\right| \\
& +\psi\left(R+\varphi(R)\left\|\Theta_{g}\right\|_{L^{1}}\right) \int_{0}^{\tilde{t}_{1}}\left\|S\left(\tilde{t}_{2}, \tau\right)-S\left(\tilde{t}_{1}, \tau\right)\right\|_{B(E)} \Theta_{f}(\tau) d \tau \\
& +\frac{M\left\|\Theta_{f}\right\|_{L^{r}} \psi\left(R+\varphi(R)\left\|\Theta_{g}\right\|_{L^{1}}\right)}{\delta^{1-\frac{1}{r}}}\left(e^{-\frac{r \delta}{r-1}\left(t-\tilde{t}_{2}\right)}-e^{-\frac{r \delta}{r-1}\left(t-\tilde{t}_{1}\right)}\right)^{1-\frac{1}{r}}
\end{aligned}
$$

Case 2. For the interval $\left(s_{i}, t_{i+1}\right], s_{i} \leq \tilde{t}_{1} \leq \tilde{t}_{2} \leq t_{i+1}$, any $y \in B_{R}$, then we get

$$
\begin{aligned}
\left|(\Lambda y)\left(\tilde{t}_{2}\right)-(\Lambda y)\left(\tilde{t}_{1}\right)\right| & \leq\left\|\frac{\partial}{\partial s} S\left(\tilde{t}_{2}, s_{i}\right)-\frac{\partial}{\partial s} S\left(\tilde{t}_{1}, s_{i}\right)\right\|_{B(E)}\left|\gamma_{i}\left(s_{i}, y\left(s_{i}\right)\right)\right| \\
& +\left\|S\left(\tilde{t}_{2}, s_{i}\right)-S\left(\tilde{t}_{1}, s_{i}\right)\right\|_{B(E)}\left|\zeta_{i}\left(s_{i}, y\left(s_{i}\right)\right)\right| \\
& +\mid \int_{s_{i}}^{\tilde{t}_{1}}\left(S\left(\tilde{t}_{2}, s\right)-S\left(\tilde{t}_{1}, s\right)\right) f\left(s, y(s), \int_{0}^{s} g(s, \tau, y(\tau)) d \tau\right) d s \\
& +\int_{\tilde{t}_{1}}^{\tilde{t}_{2}} S\left(\tilde{t}_{2}, \tau\right) f\left(s, y(s), \int_{0}^{s} g(s, \tau, y(\tau)) d \tau\right) d s \mid \\
& \leq \int_{s_{i}}^{\tilde{t}_{1}}\left\|S\left(\tilde{t}_{2}, \tau\right)-S\left(\tilde{t}_{1}, \tau\right)\right\|_{B(E)} \Theta_{f}(\tau) \psi\left(|y(s)|+\int_{0}^{s} \Theta_{g}(\tau) \varphi(|y(\tau)|) d \tau\right) d s \\
& +M \int_{\tilde{t}_{1}}^{\tilde{t}_{2}} \Theta_{f}(s) \psi\left(|y(s)|+\int_{0}^{s} \Theta_{g}(\tau) \varphi(|y(\tau)|) d \tau\right) d s
\end{aligned}
$$

It follows from the Hölder's inequality that 


$$
\begin{aligned}
\left|(\Lambda y)\left(\tilde{t}_{2}\right)-(\Lambda y)\left(\tilde{t}_{1}\right)\right| & \leq\left\|\frac{\partial}{\partial s} S\left(\tilde{t}_{2}, s_{i}\right)-\frac{\partial}{\partial s} S\left(\tilde{t}_{1}, s_{i}\right)\right\|_{B(E)}\left|\gamma_{i}\left(s_{i}, y\left(s_{i}\right)\right)\right| \\
& +\left\|S\left(\tilde{t}_{2}, s_{i}\right)-S\left(\tilde{t}_{1}, s_{i}\right)\right\|_{B(E)}\left|\zeta_{i}\left(s_{i}, y\left(s_{i}\right)\right)\right| \\
& +\psi\left(R+\varphi(R)\left\|\Theta_{g}\right\|_{L^{1}}\right) \int_{s_{i}}^{\tilde{t}_{1}}\left\|S\left(\tilde{t}_{2}, \tau\right)-S\left(\tilde{t}_{1}, \tau\right)\right\|_{B(E)} p(\tau) d \tau \\
& +\frac{M\left\|\Theta_{f}\right\|_{L^{r}} \psi\left(R+\varphi(R)\left\|\Theta_{g}\right\|_{L^{1}}\right)}{\delta^{1-\frac{1}{r}}}\left(e^{-\frac{r \delta}{r-1}\left(t-\tilde{t}_{2}\right)}-e^{-\frac{r \delta}{r-1}\left(t-\tilde{t}_{1}\right)}\right)^{1-\frac{1}{r}} .
\end{aligned}
$$

Case 3. For the interval $\left(s_{i}, t_{i}\right], s_{i} \leq \tilde{t}_{1} \leq \tilde{t}_{2} \leq t_{i}$, any $y \in B_{R}$, we have

$$
\begin{aligned}
& \left|(\Lambda y)\left(\tilde{t}_{2}\right)-(\Lambda y)\left(\tilde{t}_{1}\right)\right| \\
& =\mid \gamma_{i}\left(\tilde{t}_{2},-\frac{\partial}{\partial s} S\left(\tilde{t}_{2}, s_{i}\right) \gamma_{i-1}\left(s_{i-1}, y\left(t_{i-1}^{-}\right)\right)+S\left(\tilde{t}_{2}, s_{i-1}\right) \zeta_{i-1}\left(s_{i-1}, y\left(t_{i-1}^{-}\right)\right)\right. \\
& \left.+\int_{s_{i-1}}^{\tilde{t}_{2}} S(t, s) f\left(s, y(s), \int_{0}^{s} g(s, \tau, y(\tau)) d \tau\right) d s\right) \\
& -\gamma_{i}\left(\tilde{t}_{1},-\frac{\partial}{\partial s} S\left(\tilde{t}_{1}, s_{i}\right) \gamma_{i-1}\left(s_{i-1}, y\left(t_{i-1}^{-}\right)\right)+S\left(\tilde{t}_{1}, s_{i-1}\right) \zeta_{i-1}\left(s_{i-1}, y\left(t_{i-1}^{-}\right)\right)\right. \\
& \left.+\int_{s_{i-1}}^{\tilde{t}_{1}} S\left(\tilde{t}_{1}, s\right) f\left(s, y(s), \int_{0}^{s} g(s, \tau, y(\tau)) d \tau\right) d s\right) \mid .
\end{aligned}
$$

then

$$
\begin{aligned}
& \left|(\Lambda y)\left(\tilde{t}_{2}\right)-(\Lambda y)\left(\tilde{t}_{1}\right)\right| \\
& \leq c_{i} \mid-\frac{\partial}{\partial s} S\left(\tilde{t}_{2}, s_{i}\right) \gamma_{i-1}\left(s_{i-1}, y\left(t_{i-1}^{-}\right)\right)+S\left(\tilde{t}_{2}, s_{i-1}\right) \zeta_{i-1}\left(s_{i-1}, y\left(t_{i-1}^{-}\right)\right) \\
& +\int_{s_{i-1}}^{\tilde{t}_{2}} S(t, s) f\left(s, y(s), \int_{0}^{s} g(s, \tau, y(\tau)) d \tau\right) d s \\
& +\frac{\partial}{\partial s} S\left(\tilde{t}_{1}, s_{i}\right) \gamma_{i-1}\left(s_{i-1}, y\left(t_{i-1}^{-}\right)\right)-S\left(\tilde{t}_{1}, s_{i-1}\right) \zeta_{i-1}\left(s_{i-1}, y\left(t_{i-1}^{-}\right)\right) \\
& -\int_{s_{i-1}}^{\tilde{t}_{1}} S\left(\tilde{t}_{1}, s\right) f\left(s, y(s), \int_{0}^{s} g(s, \tau, y(\tau)) d \tau\right) d s \mid
\end{aligned}
$$

Similarly, one can easily see that

$$
\begin{aligned}
\left|(\Lambda y)\left(\tilde{t}_{2}\right)-(\Lambda y)\left(\tilde{t}_{1}\right)\right| & \leq c_{i}\left\|\frac{\partial}{\partial s} S\left(\tilde{t}_{2}, s_{i-1}\right)-\frac{\partial}{\partial s} S\left(\tilde{t}_{1}, s_{i-1}\right)\right\|_{B(E)}\left|\gamma_{i-1}\left(s_{i-1}, y\left(t_{i-1}^{-}\right)\right)\right| \\
& +c_{i}\left\|S\left(\tilde{t}_{2}, s_{i-1}\right)-S\left(\tilde{t}_{1}, s_{i-1}\right)\right\|_{B(E)}\left|\zeta_{i-1}\left(s_{i-1}, y\left(t_{i-1}^{-}\right)\right)\right| \\
& +c_{i} \psi\left(R+\varphi(R)\left\|\Theta_{g}\right\|_{L^{1}}\right) \int_{s_{i-1}}^{\tilde{t}_{1}}\left\|S\left(\tilde{t}_{2}, \tau\right)-S\left(\tilde{t}_{1}, \tau\right)\right\|_{B(E)} \Theta_{f}(\tau) d \tau \\
& +\frac{M c_{i}\left\|\Theta_{f}\right\|_{L^{r}} \psi\left(R+\varphi(R)\left\|\Theta_{g}\right\|_{L^{1}}\right)}{\delta^{1-\frac{1}{r}}}\left(e^{-\frac{r \delta}{r-1}\left(t-\tilde{t}_{2}\right)}-e^{-\frac{r \delta}{r-1}\left(t-\tilde{t}_{1}\right)}\right)^{1-\frac{1}{r}} .
\end{aligned}
$$

In view of Case $1-3$, as a result, $\left\|(\Lambda y)\left(\tilde{t}_{2}\right)-(\Lambda y)\left(\tilde{t}_{1}\right)\right\| \rightarrow 0$ as $\tilde{t}_{2} \rightarrow \tilde{t}_{1}$, which meansthat $\Lambda$ is equicontinuous.

Step 4. $\Lambda$ is a $\alpha_{P C}$-contraction operator.

For every bounded subset $B \subset P C(J, E)$, then we know that there exists a countable set $B_{1}=$ $\{y\}_{n=1}^{\infty} \subset B$ (see Lemma 2), such that for any $t \in J$, we have

$$
\alpha_{E}(\Lambda(B)(t)) \leq 2 \alpha_{E}\left(\Lambda\left(B_{1}\right)(t)\right) .
$$


Note that $B$ and $\Lambda B$ are equicontinuous, we can get from Lemma 2, Lemma 3, Lemma 4 and using the assumptions $\left(H_{1}\right)-\left(H_{6}\right)$, we obtain

Case 1. For the interval $\left(t_{i}, s_{i}\right]$, we have

$$
\begin{aligned}
& \alpha_{E}\left(\Lambda B_{1}(t)\right) \leq \tilde{M} k_{i}\left\{\alpha_{E}\left(\gamma_{i-1}\left(s_{i-1}, y_{n}\left(t_{i-1}^{-}\right)\right)\right)\right\}_{n=0}^{\infty} \\
& +M k_{i}\left\{\alpha_{E}\left(\zeta_{i-1}\left(s_{i-1}, y_{n}\left(t_{i-1}^{-}\right)\right)\right)\right\}_{n=0}^{\infty=0} \\
& +k_{i} \alpha_{E}\left(\left\{\int_{s_{i-1}}^{t} S(t, s) f\left(s, y_{n}(s), \int_{0}^{s} g\left(s, \tau, y_{n}(s)\right) d \tau\right) d s\right\}_{n=0}^{\infty}\right) \\
& \left.\left.\leq \tilde{M} k_{i} k_{i-1}\left\{\alpha_{E}\left(y_{n}\left(t_{i}^{-}\right)\right)\right\}_{n=0}^{\infty}\right)+M k_{i} \bar{k}_{i-1}\left\{\alpha_{E}\left(y_{n}\left(t_{i-1}^{-}\right)\right)\right\}_{n=0}^{\infty}\right) \\
& \left.+2 M k_{i} \int_{s_{i-1}}^{t}\left\{\alpha_{E}\left(f\left(s, y_{n}(s), \int_{0}^{s} g\left(s, \tau, y_{n}(\tau)\right) d \tau\right) d s\right)\right)\right\}_{n=0}^{\infty} d s \\
& \left.\left.\leq \tilde{M} k_{i} k_{i-1}\left\{\alpha_{E}\left(y_{n}\left(t_{i}^{-}\right)\right)\right\}_{n=0}^{\infty}\right)+M k_{i} \bar{k}_{i-1}\left\{\alpha_{E}\left(y_{n}\left(t_{i-1}^{-}\right)\right)\right\}_{n=0}^{\infty}\right) \\
& +2 M k_{i} \int_{s_{i-1}}^{t} \sigma_{1}(s)\left\{\alpha_{E}\left(y_{n}(s)\right)\right\}_{n=0}^{\infty} \\
& \left.+\varrho_{i}(s)\left\{\alpha_{E}\left(\int_{0}^{s} g\left(s, \tau, y_{n}(\tau)\right) d \tau\right)\right\}_{n=0}^{\infty}\right) d s \\
& \left.\left.\leq \tilde{M} k_{i} k_{i-1}\left\{\alpha_{E}\left(y_{n}\left(t_{i}^{-}\right)\right)\right\}_{n=0}^{\infty}\right)+M k_{i} \bar{k}_{i-1}\left\{\alpha\left(y_{n}\left(t_{i-1}^{-}\right)\right)\right\}_{n=0}^{\infty}\right) \\
& \left.+2 M k_{i} \int_{s_{i-1}}^{t} \sigma_{i}(s)\left\{\alpha_{E}\left(y_{n}(s)\right)\right\}_{n=0}^{\infty}\right) \\
& \left.+2 K^{*} \varrho_{i}(s)\left\{\int_{0}^{s} \alpha_{E}\left(y_{n}(\tau)\right) d \tau\right\}_{n=0}^{\infty}\right) d s \\
& \leq\left(\tilde{M} k_{i} k_{i-1}+M k_{i} \bar{k}_{i-1}\right) \alpha_{E}\left(B_{1}\left(t_{i}^{-}\right)\right) \\
& +2 M k_{i} \int_{s_{i-1}}^{t}\left(\sigma_{i}(s) \alpha_{E}\left(B_{1}(s)\right)+2 K^{*} \varrho_{i}(s) \int_{0}^{s} \alpha_{E}\left(B_{1}(\tau) d \tau\right) d s .\right. \\
& \leq\left(\tilde{M} k_{i} k_{i-1}+k_{i} \bar{k}_{i-1}\right) \sup _{s \in\left(t_{i}, s i\right]} \alpha_{E}(B(t)) \\
& +2 M k_{i} \int_{s_{i-1}}^{t}\left(\sigma(s) \alpha_{E}\left(B_{1}(s)\right)+2 K^{*} \varrho(s) s \sup _{\tau \in[0, s]} \alpha_{E}\left(B_{1}(\tau)\right) d s .\right. \\
& \leq\left(\tilde{M} k_{i} k_{i-1}+M k_{i} \bar{k}_{i-1}\right) \sup _{s \in\left(s_{i}, t_{i+1}\right]} \alpha_{E}(B(t)) \\
& +2 M \int_{s_{i-1}}^{t}\left(\sigma(s) \sup _{s \in\left(s_{i}, t_{i+1}\right]} \alpha_{E}\left(B_{1}(s)\right)+2 K^{*} \varrho(s) s \sup _{\tau \in\left(t_{i}, s_{i}\right]} \alpha_{E}\left(B_{1}(\tau)\right) d s\right. \text {. } \\
& \leq\left(\tilde{M} k_{i} k_{i-1}+M k_{i} \bar{k}_{i-1}\right) \sup _{s \in\left(t_{i}, s_{i}\right]} \alpha_{E}(B(t)) \\
& +2 M k_{i} \int_{s_{i-1}}^{t}\left(\sigma(s)+2 K^{*} s \varrho(s)\right) \sup _{s \in\left(t_{i}, s_{i}\right]} \alpha_{E}\left(B_{1}(s)\right) d s \\
& \leq k_{i}\left(\tilde{M} k_{i-1}+M \bar{k}_{i-1}+2 M\left(\|\sigma\|_{L^{1}}+2 K^{*} s_{i}\|\varrho\|_{L^{1}}\right)\right) \sup _{t \in\left(t_{i}, s_{i}\right]} \alpha_{E}(B(t)) \\
& \leq k_{i}\left(\tilde{M} k_{i-1}+M \bar{k}_{i-1}+2 M\left(\|\sigma\|_{L^{1}}+2 K^{*} a\|\varrho\|_{L^{1}}\right) \sup _{t \in\left(t_{i}, s_{i}\right]} \alpha_{E}(B(t))\right. \text {. }
\end{aligned}
$$

Then

$$
\alpha_{E}(N(B(t))) \leq k_{i}\left(\tilde{M} k_{i-1}+M \bar{k}_{i-1}+2 M\left(\|\sigma\|_{L^{1}}+2 K^{*} a\|\varrho\|_{L^{1}}\right) \alpha_{P C}(B(t)) .\right.
$$

Case 2. For the interval $\left[0, t_{1}\right]$, we have

$$
\alpha_{E}\left(\Lambda B_{1}(t)\right) \leq \alpha_{E}\left(\left\{\int_{0}^{t} S(t, s) f\left(s, y_{n}(s), \int_{0}^{s} g\left(s, \tau, y_{n}(s)\right) d \tau\right) d s\right\}_{n=0}^{\infty}\right)
$$




$$
\begin{aligned}
\leq & \left.2 M \int_{0}^{t}\left\{\alpha_{E}\left(f\left(s, y_{n}(s), \int_{0}^{s} g\left(s, \tau, y_{n}(\tau)\right) d \tau\right) d s\right)\right)\right\}_{n=0}^{\infty} d s \\
\leq & 2 M \int_{0}^{t} \sigma_{1}(s)\left\{\alpha_{E}\left(y_{n}(s)\right)\right\}_{n=0}^{\infty} d s \\
& \left.+\varrho_{i}(s)\left\{\alpha_{E}\left(\int_{0}^{s} g\left(s, \tau, y_{n}(\tau)\right) d \tau\right)\right\}_{n=0}^{\infty}\right) d s \\
\leq & \left.2 M \int_{0}^{t} \sigma_{i}(s)\left\{\alpha_{E}\left(y_{n}(s)\right)\right\}_{n=0}^{\infty}\right) \\
& \left.+2 K^{*} \varrho_{i}(s)\left\{\int_{0}^{s} \alpha_{E}\left(y_{n}(\tau)\right) d \tau\right\}_{n=0}^{\infty}\right) d s \\
\leq & 2 M \int_{0}^{t}\left(\sigma_{i}(s) \alpha_{E}\left(B_{1}(s)\right)+2 K^{*} \varrho_{i}(s) \int_{0}^{s} \alpha_{E}\left(B_{1}(\tau) d \tau\right) d s\right. \\
\leq & 2 M \int_{0}^{t}\left(\sigma(s) \alpha_{E}\left(B_{1}(s)\right)+2 K^{*} \varrho(s) s \sup _{\tau \in[0, s]} \alpha_{E}\left(B_{1}(\tau)\right) d s\right. \\
\leq & 2 M \int_{0}^{t}\left(\sigma(s) \sup _{s \in\left[0 ; t_{1}\right]} \alpha_{E}\left(B_{1}(s)\right)+2 K^{*} \varrho(s) s \sup _{\tau \in\left[0 ; t_{1}\right]} \alpha_{E}\left(B_{1}(\tau)\right) d s\right. \\
\leq & 2 M \int_{0}^{t}\left(\sigma(s)+2 K^{*} s \varrho(s)\right) \sup _{s \in\left[0 ; t_{1}\right]} \alpha_{E}\left(B_{1}(s)\right) d s . \\
\leq & 2 M\left(\|\sigma\|_{L^{1}}+2 K^{*} t_{1}\|\varrho\|_{L^{1}}\right) \sup _{t \in\left[0 ; t_{1}\right]} \alpha_{E}(B(t)) \\
\leq & 2 M\left(\|\sigma\|_{L^{1}}+2 K^{*} a\|\varrho\|_{L^{1}}\right) \sup _{t \in\left[0 ; t_{1}\right]} \alpha_{E}(B(t)) . \\
& \\
\leq &
\end{aligned}
$$

Then

$$
\left.\alpha_{E}(\Lambda(B(t))) \leq 2 M\left(\|\sigma\|_{L^{1}}+2 K^{*} a\|\varrho\|_{L^{1}}\right)\right) \alpha_{P C}(B(t)) .
$$

Case 3. For the interval $\left(s_{i}, t_{i+1}\right]$, we have

$$
\begin{aligned}
\alpha_{E}\left(\Lambda B_{1}(t)\right) \leq & \tilde{M}\left\{\alpha_{E}\left(\gamma_{i}\left(s, y_{n}\left(t_{i}^{-}\right)\right)\right)\right\}_{n=0}^{\infty}+M\left\{\alpha_{E}\left(\zeta_{i}\left(s, y_{n}\left(t_{i}^{-}\right)\right)\right)\right\}_{n=0}^{\infty} \\
& +\alpha_{E}\left(\left\{\int_{s_{i}}^{t} s(t, s) f\left(s, y_{n}(s), \int_{0}^{s} g\left(s, \tau, y_{n}(s)\right) d \tau\right) d s\right\}_{n=0}^{\infty}\right) \\
& \left.\left.\leq \tilde{M} k_{i}\left\{\alpha_{E}\left(y_{n}\left(t_{i}^{-}\right)\right)\right\}_{n=0}^{\infty}\right)+M \bar{k}_{i}\left\{\alpha_{E}\left(y_{n}\left(t_{i}^{-}\right)\right)\right\}_{n=0}^{\infty}\right) \\
& \left.+2 M \int_{s_{i}}^{t}\left\{\alpha_{E}\left(f\left(s, y_{n}(s), \int_{0}^{s} g\left(s, \tau, y_{n}(\tau)\right) d \tau\right) d s\right)\right)\right\}_{n=0}^{\infty} d s \\
& \left.\left.\leq \tilde{M} k_{i}\left\{\alpha_{E}\left(y_{n}\left(t_{i}^{-}\right)\right)\right\}_{n=0}^{\infty}\right)+M \bar{k}_{i}\left\{\alpha_{E}\left(y_{n}\left(t_{i}^{-}\right)\right)\right\}_{n=0}^{\infty}\right) \\
& +2 M \int_{s_{i}}^{t} \sigma_{1}(s)\left\{\alpha_{E}\left(y_{n}(s)\right)\right\}_{n=0}^{\infty} d s \\
& \left.+\varrho_{i}(s)\left\{\alpha_{E}\left(\int_{0}^{s} g\left(s, \tau, y_{n}(\tau)\right) d \tau\right)\right\}_{n=0}^{\infty}\right) d s
\end{aligned}
$$




$$
\begin{aligned}
& \left.\left.\leq \tilde{M} k_{i}\left\{\alpha_{E}\left(y_{n}\left(t_{i}^{-}\right)\right)\right\}_{n=0}^{\infty}\right)+M \bar{k}_{i}\left\{\alpha_{E}\left(y_{n}\left(t_{i}^{-}\right)\right)\right\}_{n=0}^{\infty}\right) \\
& \left.+2 M \int_{s_{i}}^{t} \sigma_{i}(s)\left\{\alpha_{E}\left(y_{n}(s)\right)\right\}_{n=0}^{\infty}\right) \\
& \left.+2 K^{*} \varrho_{i}(s)\left\{\int_{0}^{s} \alpha_{E}\left(y_{n}(\tau)\right) d \tau\right\}_{n=0}^{\infty}\right) d s \\
& \leq\left(\tilde{M} k_{i}+M \bar{k}_{i}\right) \alpha_{E}\left(B\left(t_{i}^{-}\right)\right) \\
& +2 M \int_{s_{i}}^{t}\left(\sigma(s) \alpha_{E}\left(B_{1}(s)\right)+2 K^{*} \varrho(s) \int_{0}^{s} \alpha_{E}\left(B_{1}(\tau) d \tau\right) d s\right. \\
& \leq\left(\tilde{M} k_{i}+M \bar{k}_{i}\right) \sup _{s \in\left(s_{i}, t_{i+1}\right]} \alpha_{E}\left(B_{1}(s)\right) \\
& +2 M \int_{s_{i}}^{t}\left(\sigma(s) \alpha_{E}\left(B_{1}(s)\right)+2 K^{*} \varrho(s) s \sup _{\tau \in[0, s]} \alpha_{E}\left(B_{1}(\tau)\right) d s\right. \\
& \leq\left(\tilde{M} k_{i}+M \bar{k}_{i}\right) \sup _{s \in\left(s_{i}, t_{i+1}\right]} \alpha_{E}(B(s)) \\
& +2 M \int_{s_{i}}^{t}\left(\sigma(s) \sup _{s \in\left(s_{i}, t_{i+1}\right]} \alpha_{E}\left(B_{1}(s)\right)+2 K^{*} \varrho(s) s \sup _{\tau \in\left(s_{i}, t_{i+1}\right]} \alpha_{E}\left(B_{1}(\tau)\right) d s\right. \\
& \leq\left(\tilde{M} k_{i}+M \bar{k}_{i}\right) \alpha_{E}\left(B_{1}\left(t_{i}^{-}\right)\right) \\
& +2 M \int_{s_{i}}^{t}\left(\sigma(s)+2 K^{*} s \varrho(s)\right) \sup _{s \in\left(s_{i}, t_{i+1}\right]} \alpha_{E}\left(B_{1}(s)\right) d s \text {. } \\
& \leq\left(\tilde{M} k_{i}+M \bar{k}_{i}+2 M\left(\|\sigma\|_{L^{1}}+2 K^{*} t_{i+1}\|\varrho\|_{L^{1}}\right)\right) \sup _{t \in\left(s_{i}, t_{i+1}\right]} \alpha_{E}(B(t)) \\
& \leq\left(\tilde{M} k_{i}+M \bar{k}_{i}+2 M\left(\|\sigma\|_{L^{1}}+2 K^{*} a\|\varrho\|_{L^{1}}\right)\right) \sup _{t \in\left(s_{i}, t_{i+1}\right]} \alpha_{E}(B(t)) .
\end{aligned}
$$

Then

$$
\alpha_{E}(\Lambda(B(t))) \leq\left(\tilde{M} k_{i}+M \bar{k}_{i}+2 M\left(\|\sigma\|_{L^{1}}+2 K^{*} a\|\varrho\|_{L^{1}}\right)\right) \alpha_{P C}(B(t)) .
$$

From the above cases (10)-(12), for all $t \in J$, we obtain

$$
\left.\alpha_{P C}(\Lambda(B)) \leq \max _{1 \leq i \leq N}\left(k_{i}, 1\right)\left(\max _{1 \leq i \leq N}\left(\tilde{M} k_{i}+M \bar{k}_{i}\right)+2 M\left(\|\sigma\|_{L^{1}}+2 K^{*} a\|\varrho\|_{L^{1}}\right)\right)\right) \alpha_{P C}(B) .
$$

Thus, we find that $\Lambda$ is $\alpha_{P C}$-contraction operator. Applying now theorem 1 , we conclude that $\Lambda$ has a fixed point which is an solution of the system (1).

Next, we present another existence result for the mild solution of the system (1).

Theorem 4. Assume that hypotheses $\left(H_{1}\right)-\left(H_{6}\right)$ are fulfilled and

$$
\lim _{R \rightarrow+\infty} \inf \frac{\left.\psi\left(R+\left\|\Theta_{g}\right\|_{L^{1}} \varphi(R)\right)\left\|\Theta_{f}\right\|_{L^{r}}\right)}{R}=\rho<\infty,
$$

and

$$
\tilde{M} c_{i}+M e_{i}+\frac{M \rho\left\|\Theta_{f}\right\|_{L^{r}}}{\delta^{1-\frac{1}{r}}} \leq 1, i=1, \cdots, N .
$$

Then, there exists a mild solution of system (1).

Proof. Following the proof of Theorem 3 we conclude that the map $\Lambda: B_{R} \rightarrow B_{R}$ given by Equation (5) is continuous. Next, we show that there exists $R>0$ such that $\Lambda\left(B_{R}\right) \subset B_{R}$. In fact, if it is not true, 
then for each positive number $R$, there exists a function $\check{y} \in B_{R}$ and $\check{t} \in J$ such that $R \leq|(\Lambda y)(\check{t})|$. Therefore for

Case 1 . For $\check{t} \in\left(s_{i}, t_{i}\right]$, and $\check{y} \in B_{R}$, we have,

$$
\begin{aligned}
|(\Lambda \check{y})(\check{t})| & \leq\left\|\frac{\partial}{\partial s} S\left(\breve{t}, s_{i-1}\right)\right\|_{B(E)}\left|\gamma_{i}\left(s_{i-1}, \check{y}\left(s_{i-1}\right)\right)\right| \\
& +\left\|S\left(\check{t}, s_{i-1}\right)\right\|_{B(E)}\left|\zeta_{i}\left(s_{i-1}, \check{y}\left(s_{i-1}\right)\right)\right| \\
& \left.+\int_{s_{i-1}}^{\check{t}}|| S(t, s) \|_{B(E)} \Theta_{f}(s) \psi\left(|\check{y}(s)|+\int_{0}^{s} \Theta_{g}(\tau) \varphi(|\check{y}(\tau)|)\right) d \tau\right) d s \\
& \leq \tilde{M} c_{i-1}\left|\check{y}\left(s_{i-1}\right)\right|+\tilde{M} d_{i-1} \\
& +M e_{i-1}\left|\check{y}\left(s_{i-1}\right)\right|+M l_{i-1} \\
& +\int_{s_{i-1}}^{\check{t}} M e^{-\delta(t-s)} \Theta_{f}(s) \psi\left(|\check{y}(s)|+\int_{0}^{s} \Theta_{g}(\tau) \varphi(|\check{y}(\tau)|) d \tau\right) d s .
\end{aligned}
$$

Then

$$
\begin{aligned}
|(\Lambda y)(\check{t})| & \leq \tilde{M} c_{i-1} R+\tilde{M} d_{i-1} \\
& +M e_{i-1} R+M l_{i-1} \\
& +\int_{s_{i-1}}^{\check{t}} M e^{-\delta(t-s)} \Theta_{f}(s) \psi\left(|\check{y}(s)|+\int_{0}^{s} \Theta_{g}(\tau) \varphi(|\check{y}(\tau)|) d \tau\right) d s . \\
& \leq\left(\tilde{M} c_{i-1}+M e_{i-1}\right) R+\tilde{M} l_{i-1}+M l_{i-1} \\
& +M \psi\left(R+\left\|\Theta_{g}\right\|_{L^{1}} \varphi(R)\right) \int_{s_{i-1}}^{z} e^{-\delta(t-s)} \Theta_{f}(s) d s .
\end{aligned}
$$

It follows from the Hölder's inequality that

$$
\begin{aligned}
|(\Lambda y)(\check{t})| & \leq\left(\tilde{M} c_{i-1}+M e_{i-1}\right) R+\tilde{M} d_{i-1}+M l_{i-1} \\
& +\frac{M \psi\left(R+\left\|\Theta_{g}\right\|_{L^{1}} \varphi(R)\right)\left\|\Theta_{f}\right\|_{L^{r}}}{\delta^{1-\frac{1}{r}}} .
\end{aligned}
$$

Case 2. For $\check{t} \in\left[0 ; t_{1}\right]$, and $\check{y} \in B_{R}$, we get,

$$
\begin{aligned}
|(\Lambda y)(\check{t})| & \leq\left\|\frac{\partial}{\partial s} S(t, 0)\right\|_{B(E)}\left|y_{0}\right| \\
& +\|S(t, s)\|_{B(E)}\left|y_{1}\right| \\
& +\int_{0}^{\check{t}}\|S(t, s)\|_{B(E)} \Theta_{f}(s) \psi\left(|\check{y}(s)|+\int_{0}^{s} \Theta_{g}(\tau) \varphi(|\check{y}(\tau)|) d \tau\right) d s \\
& \leq \tilde{M}\left|y_{1}\right|+M\left|y_{0}\right|+M \psi\left(R+\left\|\Theta_{g}\right\|_{L^{1}} \varphi(R)\right) \int_{0}^{\check{t}} e^{-\delta(t-s)} \Theta_{f}(s) d s .
\end{aligned}
$$

It follows from the Hölder's inequality that

$$
\begin{aligned}
|(\Lambda y)(\check{t})| & \leq \tilde{M}\left|y_{0}\right|+M\left|y_{1}\right|+\frac{M \psi\left(R+\left\|\Theta_{g}\right\|_{L^{1}} \varphi(R)\right)\left\|\Theta_{f}\right\|_{L^{r}}}{\delta^{1-\frac{1}{r}}}\left(1-e^{-\frac{r \delta}{r-1} \check{t}}\right)^{1-\frac{1}{r}} \\
& \leq \tilde{M}\left|y_{0}\right|+M\left|y_{1}\right|+\frac{M \psi\left(R+\left\|\Theta_{g}\right\|_{L^{1}} \varphi(R)\right)\left\|\Theta_{f}\right\|_{L^{r}}}{\delta^{1-\frac{1}{r}}} .
\end{aligned}
$$


Case 3. For $\check{t} \in\left(s_{i}, t_{i+1}\right]$, and $\check{y} \in B_{R}$, we have,

$$
\begin{aligned}
|(\Lambda y)(\check{t})| & \leq \tilde{M} c_{i}|y(\check{t})|+\tilde{M} d_{i}+M e_{i}|y(\check{t})|+M l_{i} \\
& +\int_{s_{i}}^{t}\|S(\check{t}, s)\|_{B(E)} \Theta_{f}(s) \psi\left(|\check{y}(s)|+\int_{0}^{s} \Theta_{g}(\tau) \varphi(|\check{y}(\tau)|) d \tau\right) d s \\
& \leq \tilde{M} c_{i} R+\tilde{M} d_{i}+M e_{i} R+M l_{i} \\
& +M \psi\left(R+\left\|\Theta_{g}\right\|_{L^{1}} \varphi(R)\right) \int_{s_{i}}^{t} e^{-\delta(\check{t}-s)} \Theta_{f}(s) d s .
\end{aligned}
$$

It follows from the Hölder's inequality that

$$
\begin{aligned}
|(\Lambda y)(t)| & \leq \tilde{M} d_{i}+M l_{i} \\
& +\left(\tilde{M} c_{i}+M e_{i}\right) R \\
& +\frac{M \psi\left(R+\left\|\Theta_{g}\right\|_{L^{1}} \varphi(R)\right)\left\|\Theta_{f}\right\|_{L^{r}}}{\delta^{1-\frac{1}{r}}}
\end{aligned}
$$

Therefore for all $\breve{t} \in J$, we have

$$
\begin{aligned}
R<|(\Lambda y)(\check{t})| & \leq\left(\tilde{M} c_{i}+M e_{i}\right) R \\
& +\max \left(\tilde{M} d_{i}+M l_{i}, \tilde{M}\left|y_{0}\right|+M\left|y_{1}\right|\right) \\
& +\frac{M \psi\left(R+\left\|\Theta_{g}\right\|_{L^{1}} \varphi(R)\right)\left\|\Theta_{f}\right\|_{L^{r}}}{\delta^{1-\frac{1}{r}}}
\end{aligned}
$$

Dividing both sides by $R$ and taking the lim inf as $R \rightarrow+\infty$, we have

$$
\tilde{M} c_{i}+M e_{i}+\frac{M \rho\left\|\Theta_{f}\right\|_{L^{r}}}{\delta^{1-\frac{1}{r}}}>1, i=0, \cdots, N .
$$

which contradicts (13). Hence, the operator $\Lambda$ transforms the set $B_{R}$ into itself.

The proof of $\Lambda: B_{R} \rightarrow B_{R}$ is $\alpha_{E}$-contraction is similar to those in Theorem 3. Therefore, we omit the details. By the Darbo-Sadovskii fixed point theorem 2 we deduce that $\Lambda$ has a fixed point which is a mild solution of system (1).

\section{An Example}

In this section, we give an example to illustrate the above theoretical result.

Set $E=L^{2}([0, \pi], \mathbb{R})$ be the space of all square integrable functions from $[0, \pi]$ into $\mathbb{R}$. We denote by $\mathbb{H}^{2}([0, \pi], \mathbb{R})$ the Sobolev space of functions $u:[0, \pi] \rightarrow \mathbb{R}$, such that $u^{\prime \prime} \in L^{2}([0, \pi], \mathbb{R})$. Define the operator $\mathbb{A}: D(\mathbb{A}) \rightarrow E$ by

$$
\mathbb{A} u(\tau)=u^{\prime \prime}(\tau)
$$

with domain

$$
D(\mathbb{A})=\left\{\omega \in E: \omega, \omega^{\prime} \text { are absolutely continuous, } \omega^{\prime \prime} \in E, \omega(0)=\omega(\pi)=0\right\} .
$$

It is well known that $\mathbb{A}$ is the infinitesimal generator of a $C_{0}$-semigroup and of a strongly continuous cosine function on $E$, which will be denoted by $(C(t))$. From [14], for all $x \in$ $\mathbb{H}^{2}([0, \pi], \mathbb{R}), t \in \mathbb{R},\|C(t)\|_{B(E)} \leq 1$. Define also the operator $\mathbb{B}: \mathbb{H}^{1}([0, \pi], \mathbb{R}) \rightarrow E$ by

$$
\mathbb{B}(t) u(s)=a(t) u^{\prime}(s),
$$

where $a:[0,1] \rightarrow \mathbb{R}$ is a Hölder continuous function. 
Consider the closed linear operator $\mathcal{A}(t)=\mathbb{B}(t)+\mathbb{A}$. It has been proved by Henríquez in [44] that the family $\{\mathcal{A}(t): t \in J\}$ generates an evolution operator $\{S(t, s)\}_{(t, s) \in D}$. Moreover, $S(\cdot, \cdot)$ is well defined and satisfies the conditions (H1) and (H2), with $M=\tilde{M}=1$ and $\delta=1$.

We consider the following system:

$$
\left\{\begin{array}{rlrl}
\frac{\partial^{2}}{\partial t^{2}} u(t, \tau) & =\frac{\partial^{2}}{\partial \tau^{2}} u(t, \tau)+a(t) \frac{\partial}{\partial t} u(t, \tau) & & \\
& +\frac{u(t, \tau)}{12(\sqrt{t}+1)(1+|u(t, \tau)|)} \sqrt{t} u(s, \tau) & & \\
& +\frac{e^{-t}}{(\sqrt{t}+1)(t+1)} \int_{0}^{t} \frac{s^{2}}{8(1+t)\left(1+u^{2}(s, \tau)\right)}, s & & t \in\left(0, \frac{1}{\sqrt{3}}\right] \cup\left(\frac{2}{\sqrt{3}}, 1\right], \\
u(t, \tau) & =\frac{1}{12} \cos \pi t u\left(\frac{1}{\sqrt{3}}-\tau\right), & & t \in\left(\frac{1}{\sqrt{3}}, \frac{2}{\sqrt{3}}\right], \tau \in[0, \pi], \\
\frac{\partial}{\partial t} u(t, \tau) & =\frac{1}{12} \sin \pi t u\left(\frac{1}{\sqrt{3}}, \tau\right), & & t \in\left(\frac{1}{\sqrt{3}}, \frac{2}{\sqrt{3}}\right], \tau \in[0, \pi], \\
u(t, 0)= & u(t, \pi)=0, & t \in[0,1], \\
u(0, \tau) & =y_{0}, & & \tau \in[0, \pi], \\
\frac{\partial}{\partial t} u(0, \tau) & =y_{1}, & \tau \in[0, \pi] .
\end{array}\right.
$$

Take $a=t_{2}=1, t_{0}=s_{0}=0, t_{1}=\frac{1}{\sqrt{3}}, s_{1}=\frac{2}{\sqrt{3}}$. The system (14) can be written in the abstract form:

$$
\left\{\begin{array}{l}
y^{\prime \prime}(t)=A(t) y(t)+f\left(t, y(t), \int_{0}^{t} g(t, s, y(s)) d s\right), t \in\left(s_{i}, t_{i+1}\right], i=1,2 \\
y(t)=\gamma_{i}\left(t, y\left(t_{i}^{-}\right)\right), \quad t \in\left(t_{i}, s_{i}\right], \quad i=1, \\
y^{\prime}(t)=\zeta_{i}\left(t, y\left(t_{i}^{-}\right)\right), \quad t \in\left(t_{i}, s_{i}\right], \quad i=1 \\
y(0)=y_{0}, y^{\prime}(0)=y_{1},
\end{array}\right.
$$

where $y(t)=u(t, \cdot)$, that is $y(t)(\tau)=u(t, \tau), \tau \in[0, \pi]$.

The function $f: J \times E \times E \rightarrow E$, is given by

$$
f(t, y, z)(\tau)=\frac{|y(t)(\tau)|}{12(\sqrt{t}+1)(1+|y(t)(\tau)|)}+\frac{e^{-t}}{(\sqrt{t}+1)(t+1)} z(t)(\tau)
$$

The function $g: D \times E \rightarrow E$, is given by

$$
g(t, s, y)(\tau)=\frac{\sqrt{t} y(t)(\tau)}{8\left(1+s^{2}+t\right)\left(1+y^{2}(t)(\tau)\right)}
$$

Functions

$$
\gamma_{1}\left(t, y\left(t_{1}^{-}\right)\right)(\tau)=\frac{1}{12} \cos \pi t y\left(\frac{1}{\sqrt{3}}^{-}\right)(\tau)
$$

and

$$
\zeta_{1}\left(t, y\left(t_{1}^{-}\right)\right)(\tau)=\frac{1}{12} \sin \pi t y\left(\frac{1}{\sqrt{3}}^{-}\right)(\tau)
$$

represent noninstantaneous impulses during interval $\left(\frac{1}{\sqrt{3}}, \frac{2}{\sqrt{3}}\right]$. We have

$$
|f(t, y, z)(\tau)| \leq \frac{1}{1+\sqrt{t}} \psi(|y(t)(\tau)|+|z(t)(\tau)|)
$$

and

$$
|g(t, s, y)(\tau)| \leq \frac{\sqrt{t}}{8+8 t}|y(t)(\tau)|
$$


From the above discussion, we obtain

$$
\psi(t)=t, \varphi(t)=t, \quad \Theta_{f}(t)=\frac{1}{1+\sqrt{t}}, \quad \Theta_{g}(t)=\frac{\sqrt{t}}{8+8 t} .
$$

For each $t \in J$, and $W_{1}, W_{2} \subset E$, we get

$$
\alpha_{E}\left(f\left(t, W_{1}, W_{2}\right)\right) \leq \frac{1}{12(\sqrt{t}+1)} \alpha_{E}\left(W_{1}\right)+\frac{e^{-t}}{(\sqrt{t}+1)(t+1)} \alpha_{E}\left(W_{2}\right),
$$

We shall show that condition $\left(H_{3}\right)$ holds with

$$
\sigma(t)=\frac{1}{12(\sqrt{t}+1)}, \quad \rho(t)=\frac{e^{-t}}{(\sqrt{t}+1)(t+1)} .
$$

Moreover

$$
\|\sigma\|_{L^{1}} \leq \frac{1}{12}, \quad\|\rho\|_{L^{1}} \leq 1
$$

By (19), for any $t \in J$ and $W \subset E$, we get

$$
\alpha_{E}(g(t, s, W)) \leq \frac{1}{8} \sup _{t \in[0,1]} \frac{\sqrt{t}}{1+t} \alpha_{E}(W)
$$

then

$$
\alpha_{E}(g(t, s, W)) \leq \frac{\sqrt{2}}{24} \alpha_{E}(W)
$$

Hence $\left(H_{5}\right)$ is satisfied with $K^{*}=\frac{\sqrt{2}}{24}$.

Next, let us observe that, in view of (16) and (17), the mapping $\gamma_{1}$ and $\zeta_{1}$ fulfil the hypothes $\left(H_{5}\right)$ and $\left(H_{6}\right)$ with $c_{1}=e_{1}=k_{1}=\bar{k}_{1}=\frac{1}{12}$ and $d_{1}=l_{1}=0$. Furthermore, we have

$$
\max \left(k_{1}, 1\right)\left(\tilde{M} k_{1}+M \bar{k}_{1}+2 M\left(\|\sigma\|_{L^{1}}+2 K^{*} a\|\varrho\|_{L^{1}}\right)\right)=\frac{2+\sqrt{2}}{6}<1 .
$$

Clearly all the conditions of theorem 3 are satisfied. Hence by the conclusion of Theorem 3 , it follows that problem (14) has a solution.

Author Contributions: Investigation, M.B. and Y.Z.; Writing original draft, N.R. and B.S.

Funding: The work is supported by the Macau Science and Technology Development Fund (Grant No. 0074/2019/A2) from the Macau Special Administrative Region of the People's Republic of China and the National Natural Science Foundation of China (No. 11671339).

Acknowledgments: Y. Zhou was supported by the Macau Science and Technology Development Fund (Grant No. 0074/2019/A2) from the Macau Special Administrative Region of the People's Republic of China and the National Natural Science Foundation of China (No. 11671339). B. Samet was supported by Researchers Supporting Project RSP-2019/4, King Saud University, Saudi Arabia, Riyadh. The authors would like to thank the editor and the reviewers for their constructive comments and suggestions.

Conflicts of Interest: The authors declare no conflict of interest.

\section{References}

1. Aviles, P.; Sandefur, J. Nolinear second order equations wtih applications to partial differential equations. J. Differ. Equ. 1985, 58, 404-427. [CrossRef]

2. Azodi, H.D.; Yaghouti, M.R. Bernoulli polynomials collocation for weakly singular Volterra integro-differential equations of fractional order. Filomat 2018, 32, 3623-3635. [CrossRef]

3. Bloom, F. Asymptotic bounds for solutions to a system of damped integro-differential equations of electromagnetic theory. J. Math. Anal. Appl. 1980, 73, 524-542. [CrossRef] 
4. Forbes, L.K.; Crozier, S.; Doddrell, D.M. Calculating current densities and fields produced by shielded magnetic resonance imaging probes. SIAM J. Appl. Math. 1997, 57, 401-425.

5. Keskin, B. Reconstruction of the Volterra-type integro-differential operator from nodal points. Bound. Value Probl. 2018, 2018. [CrossRef]

6. Kostić, M. Weyl-almost periodic solutions and asymptotically Weyl-almost periodic solutions of abstract Volterra integro-differential equations. Banach J. Math. Anal. 2019, 13, 64-90. [CrossRef]

7. Ren, Y.; Qin, Y.; Sakthivel, R. Existence results for fractional order semilinear integro-differential evolution equations with infinite delay. Integral Equ. Oper. Theory 2010, 67, 33-49. [CrossRef]

8. Wu, J. Theory and Application of Partial Functional Differential Equations; Springer: New York, NY, USA, 1996.

9. Abbas, S.; Benchohra, M. Advanced Functional Evolution Equations and Inclusions; Springer: Cham, Switzerland, 2015.

10. Balachandran, K.; Park, D.G.; Anthoni, S.M. Existence of solutions of abstract nonlinear second-order neutral functional integrodifferential equations. Comput. Math. Appl. 2003, 46, 1313-1324. [CrossRef]

11. Benchohra, M.; Ntouyas, S.K. Existence of mild solutions on noncompact intervals to second order initial value problems for a class of differential inclusions with nonlocal conditions. Comput. Math. Appl. 2000, 39, 11-18. [CrossRef]

12. Fattorini, H.O. Second Order Linear Differential Equations in Banach Spaces; North-Holland Mathematics Studies; Elsevier: Amsterdam, The Netherlands, 1985; Volume 108.

13. Mönch, $\mathrm{H}$. Boundary value problems for nonlinear ordinary differential equations of second order in Banach spaces. Nonlinear Anal. 1980, 4, 985-999. [CrossRef]

14. Travis, C.C.; Webb, G.F. Second order differential equations in Banach spaces. In Proceedings of the International Symposium on Nonlinear Equations in Abstract Spaces, Arlington, TX, USA, 8-10 June 1977; Academic Press: New York, NY, USA, 1978; pp. 331-361.

15. Kozak, M. A fundamental solution of a second-order differential equation in a Banach space. Univ. Iagel. Acta Math. 1995, 32, 275-289.

16. Batty, C.J.K.; Chill, R.; Srivastava, S. Maximal regularity for second order non-autonomous Cauchy problems. Studia Math. 2008, 189, 205-223. [CrossRef]

17. Benchohra, M.; Rezoug, N.; Zhou, Y. Semilinear mixed type integro-differential evolution equations via Kuratowski measure of noncompactness. Z. Anal. Anwend. 2019, 38, 143-156

18. Cardinali, T.; Gentili, S. An existence theorem for a non-autonomous second order nonlocal multivalued problem. Stud. Univ. Babe-Bolyai Math. 2017, 6291, 101-117. [CrossRef]

19. Faraci, F.; Iannizzotto, A. A multiplicity theorem for a perturbed second-order non-autonomous system. Proc. Edinb. Math. Soc. 2006, 49, 267-275. [CrossRef]

20. Henríquez, H.; Poblete, V.; Pozo, J. Mild solutions of non-autonomous second order problems with nonlocal initial conditions. J. Math. Anal. Appl. 2014, 412, 1064-1083. [CrossRef]

21. Mahmudov, N.I.; Vijayakumar, V.; Murugesu, R. Approximate controllability of second-order evolution differential inclusions in Hilbert spaces. Mediterr. J. Math. 2016, 13, 3433-3454. [CrossRef]

22. Winiarska, T. Evolution equations of second order with operator dependent on t. Sel. Probl. Math. Cracow Univ. Tech. 1995, 6, 299-314.

23. Hernández, E.; O’Regan, D. On a new class of abstract impulsive differential equations. Proc. Am. Math. Soc. 2013, 141, 1641-1649. [CrossRef]

24. Fečkan, M.; Wang, J. A general class of impulsive evolution equations. Topol. Methods Nonlinear Anal. 2015, 46, 915-933.

25. Abbas, S.; Benchohra, M.; Darwish, M. Some existence and stability results for abstract fractional differential inclusions with not instantaneous impulses. Math. Rep. (Bucuresti) 2017, 19, 245-262.

26. Anguraj, A.; Kanjanadevi, S. Existence results for fractional non-instantaneous impulsive integro-differential equations with nonlocal conditions. Dyn. Contin. Discret. Impuls. Syst. Ser. A Math. Anal. 2016, 23, 429-445.

27. Banas, J.; Jleli, M.; Mursaleen, M.; Samet, B.; Vetro, C. Advances in Nonlinear Analysis via the Concept of Measure of Noncompactness; Springer: Singapore, 2017.

28. Benchohra, M.; Litimein, S. Existence results for a new class of fractional integro-differential equations with state dependent delay. Mem. Differ. Equ. Math. Phys. 2018, 74, 27-38.

29. Fečkan, M.; Wang, J.; Zhou, Y. Periodic solutions for nonlinear evolution equations with non-instantaneous impulses. Nonauton. Dyn. Syst. 2014, 1, 93-101. 
30. Ganga, R.; Jaydev, D. Existence result of fractional functional integro-differential equation with not instantaneous impulse. Int. J. Adv. Appl. Math. Mech. 2014, 1, 11-21.

31. Muslim, M.; Kumar, A. Controllability of fractional differential equation of order $\alpha \in(1,2]$ with non-instantaneous impulses. Asian J. Control 2018, 20, 935-942. [CrossRef]

32. Gautam, G.; Dabas, J. Mild solution for nonlocal fractional functional differential equation with not instantaneous impulse. Int. J. Nonlinear Sci. 2016, 21, 151-160.

33. Saadati, R.; Pourhadi, E.; Samet, B. On the $\mathcal{P C}$-mild solutions of abstract fractional evolution equations with non-instantaneous impulses via the measure of noncompactness. Bound. Value Probl. 2019, 2019. [CrossRef]

34. Wang, J.; Zhou, Y.; Lin, Z. On a new class of impulsive fractional differential equations. Appl. Math. Comput. 2014, 242, 649-657. [CrossRef]

35. Banaś, J.; Goebel, K. Measures of Noncompactness in Banach Spaces; Lecture Notes in Pure and Applied Mathematics 60; Dekker: New York, NY, USA, 1980.

36. Li, K.; Peng, J.; Gao, J. Nonlocal fractional semilinear differential equations in separable Banach spaces. Electron. J. Diff. Eqns. 2013, 2013, 1-7.

37. Aissani, K.; Benchohra, M. Semilinear fractional order integro-differential equations with infinite delay in Banach spaces. Arch. Math. (Brno) 2013, 49, 105-117. [CrossRef]

38. Akhmerov, R.R.; Kamenskii, M.I.; Patapov, A.S.; Rodkina, A.E.; Sadovskii, B.N. Measures of Noncompactness an Condensing Operators; Birkhauser Verlag: Basel, Switzerland, 1992.

39. Guo, D.; Lakshmikantham, V.; Liu, X. Nonlinear Integral Equations in Abstract Spaces; Kluwer Academic Publishers Group: Dordrecht, The Netherlands, 1996.

40. Chen, P.; Li, Y. Monotone iterative technique for a class of semilinear evolution equations with nonlocal conditions. Results Math. 2013, 63, 731-744. [CrossRef]

41. Heinz, H.P. On the behaviour of measure of noncompactness with respect to differentiation and integration of rector-valued functions. Nonlinear Anal. 1983, 7, 1351-1371. [CrossRef]

42. Deimling, K. Nonlinear Functional Analysis; Springer: New York, NY, USA, 1985.

43. Agarwal, R.; Meehan, M.; O'Regan, D. Fixed point theory and applications. In Cambridge Tracts in Mathematics; Cambridge University Press: New York, NY, USA, 2001.

44. Henríquez, H. Existence of solutions of non-autonomous second order functional differential equations with infinite delay. Nonlinear Anal. 2011, 74, 3333-3352. [CrossRef]

(C) 2019 by the authors. Licensee MDPI, Basel, Switzerland. This article is an open access article distributed under the terms and conditions of the Creative Commons Attribution (CC BY) license (http://creativecommons.org/licenses/by/4.0/). 